\title{
Predictive Modeling by the Cerebellum Improves Proprioception
}

\author{
Nasir H. Bhanpuri, ${ }^{1,2,3}$ Allison M. Okamura, ${ }^{4,5}$ and Amy J. Bastian ${ }^{2,3}$ \\ Departments of ${ }^{1}$ Biomedical Engineering, and ${ }^{2}$ Neuroscience, The Johns Hopkins School of Medicine, Baltimore, Maryland 21205, ${ }^{3}$ Kennedy Krieger \\ Institute, Baltimore, Maryland 21205, ${ }^{4}$ Department of Mechanical Engineering, The Johns Hopkins University, Baltimore, Maryland 21218, and \\ ${ }^{5}$ Department of Mechanical Engineering, Stanford University, Stanford, California 94305
}

\begin{abstract}
Because sensation is delayed, real-time movement control requires not just sensing, but also predicting limb position, a function hypothesized for the cerebellum. Such cerebellar predictions could contribute to perception of limb position (i.e., proprioception), particularly when a person actively moves the limb. Here we show that human cerebellar patients have proprioceptive deficits compared with controls during active movement, but not when the arm is moved passively. Furthermore, when healthy subjects move in a force field with unpredictable dynamics, they have active proprioceptive deficits similar to cerebellar patients. Therefore, muscle activity alone is likely insufficient to enhance proprioception and predictability (i.e., an internal model of the body and environment) is important for active movement to benefit proprioception. We conclude that cerebellar patients have an active proprioceptive deficit consistent with disrupted movement prediction rather than an inability to generally enhance peripheral proprioceptive signals during action and suggest that active proprioceptive deficits should be considered a fundamental cerebellar impairment of clinical importance.
\end{abstract}

\section{Introduction}

A popular idea is that the cerebellum contributes to movement control by predicting body state (i.e., position, velocity) from a copy of motor commands (i.e., efference copy) and stored knowledge of the dynamic properties of the body (Wolpert et al., 1998; Miall et al., 2007). Such a cerebellar prediction would be essential for movement because it would reduce dependence on timedelayed feedback from peripheral sensors (Todorov and Jordan, 2002; Miall et al., 2007; Xu-Wilson et al., 2009). Disrupting cerebellar activity during reaching leads to loss of body state prediction that disrupts movement accuracy (Miall et al., 2007). This cerebellar function could also contribute to proprioception, which is our sense of limb position and movement independent of vision (Proske and Gandevia, 2009). Although proprioception is signaled by sensors in the periphery (e.g., muscle spindles, skin-stretch receptors), it is also influenced by centrally generated motor commands (Wolpert et al., 1995; Proske and Gandevia, 2009) and therefore could be influenced by cerebellar mechanisms. However, it is widely accepted that proprioception is not impaired after cerebellar damage, perhaps because propriocep-

Received Feb. 14, 2013; revised June 26, 2013; accepted July 15, 2013.

Author contributions: N.H.B., A.M.O., and A.J.B. designed research; N.H.B. performed research; N.H.B., A.M.O., and A.J.B. analyzed data; N.H.B., A.M.O., and A.J.B. wrote the paper.

This work was supported by the Johns Hopkins University, the Kennedy Krieger Institute, the National Institutes of Health (Grant\#R21 NS061189 to A.M.O., Grant\#R01 HD040289 to A.J.B., and Grant\#F31 NS070512 to N.H.B.). We thank the members of the Motion Analysis Laboratory at the Kennedy Krieger Institute for helping to coordinate experiments and for providing insightful feedback and the patients for volunteering their time to participate in this study.

The authors declare no competing financial interests.

Correspondence should be addressed to Amy J. Bastian, Kennedy Krieger Institute, 707 N. Broadway, G05, Baltimore, MD 21205. E-mail: bastian@kennedykrieger.org.

DOI:10.1523/JNEUROSCI.0784-13.2013

Copyright $\odot 2013$ the authors $\quad 0270-6474 / 13 / 3314301-06 \$ 15.00 / 0$ tion has only been tested passively (i.e., after externally driven movements; Maschke et al., 2003; Bhanpuri et al., 2012). There is evidence that cerebellar damage disrupts the perception of reaching direction during a visuomotor motor adaptation task (Synofzik et al., 2008) and force perception during movement (Bhanpuri et al., 2012). These findings could be parsimoniously explained by an underlying deficit in active proprioception, but this form of perception has not been studied in depth in this patient population.

Normally, proprioception after active movements is more precise (i.e., less variable) than after similar passive movements (Paillard and Brouchon, 1974; Adamovich et al., 1998; Fuentes and Bastian, 2010). Efference-copy-based predictions of body position could augment peripheral proprioceptive signals after self-generated movements (Adamovich et al., 1998). This contribution would be distinct from $\alpha$-gamma motor neuron modulation of muscle spindles (Paillard and Brouchon, 1974; Jones et al., 2001). If enhanced precision associated with active movement were due to cerebellar predictions, the improvement should be reduced when external forces make a movement unpredictable.

Here we show that intact cerebellar function enhances proprioception during active movements by predicting movement outcomes based on dynamic models of the arm rather than through a general heightened sensitivity of proprioceptive sensors in the active arm. We compared patients with cerebellar disease with matched controls in three psychophysical tasks designed to assess passive proprioception, active proprioception with simple dynamics, and active proprioception with complex, unpredictable dynamics. All tasks relied on proprioceptive sense without vision. We found that: (1) cerebellar patients had no deficits in passive proprioception; (2) unlike controls, cerebellar patients did not 
Table 1. Characteristics of subjects

\begin{tabular}{|c|c|c|c|c|c|c|c|}
\hline \multirow[b]{2}{*}{ Subject } & \multirow[b]{2}{*}{ Age (y) } & \multirow[b]{2}{*}{ Sex } & \multirow[b]{2}{*}{$\mathrm{DH}$} & \multirow[b]{2}{*}{ Diagnosis } & \multirow[b]{2}{*}{$\begin{array}{l}\text { Saccadic } \\
\text { dysmetria }\end{array}$} & \multicolumn{2}{|l|}{ ICARS } \\
\hline & & & & & & Total $(/ 100)$ & $\begin{array}{l}\text { Kinetic } \\
(/ 52)\end{array}$ \\
\hline CRB01 & 39 & $M$ & $\mathrm{~L}$ & SCA 8 & $Y$ & 43 & 16 \\
\hline CRBO2 & 42 & $F$ & $\mathrm{R}$ & SCA 6 & $Y$ & 50 & 25 \\
\hline CRBO3* $^{*}$ & 52 & $\mathrm{~F}$ & $\mathrm{R}$ & SCA 6 & $Y$ & 23 & 11 \\
\hline CRB04 & 55 & $M$ & $\mathrm{R}$ & Sporadic & $Y$ & 61 & 26 \\
\hline CRB05 & 57 & $M$ & $\mathrm{R}$ & SCA 6 & $Y$ & 63 & 25 \\
\hline CRB06 & 58 & $\mathrm{~F}$ & $\mathrm{R}$ & SCA 6 & $Y$ & 40 & 16 \\
\hline CRB07 & 59 & $M$ & $\mathrm{~L}$ & SCA 6 & $\mathrm{~N}$ & 12 & 5 \\
\hline CRB08 & 60 & $M$ & $\mathrm{R}$ & ADCA III & $\mathrm{N}$ & 13 & 4 \\
\hline CRB09* & 61 & $\mathrm{~F}$ & $\mathrm{R}$ & SCA 6 & $Y$ & 31 & 12 \\
\hline CRB10* & 69 & $\mathrm{~F}$ & $\mathrm{R}$ & Sporadic & $\mathrm{N}$ & 18 & 10 \\
\hline CRB11 & 70 & $\mathrm{~F}$ & $\mathrm{R}$ & Sporadic & $Y$ & 62 & 22 \\
\hline
\end{tabular}

CRB group $56.5 \pm 9.6 \quad \mathrm{M}=5 \quad \mathrm{~L}=2$

CNT group $55.1 \pm 12.5 \mathrm{M}=5 \mathrm{~L}=2$

Group data are means \pm SD. CRB, cerebellar subject; $C N T$, control subject; DH, dominant hand; SCA, spinocerebellar ataxia; Sporadic, sporadic cerebellar ataxia.

*Passive WF - active WF $\geq 0.015$ (see Results for details).

show an improvement between passive and active proprioception with simple dynamics; and (3) controls performed similarly to patients in an active proprioceptive task that had unpredictable, complex dynamics.

\section{Materials and Methods}

Subjects. We tested 11 healthy human controls and 11 human patients with cerebellar damage (Table 1). Subjects were matched for sex (5 males and 6 females in each group), age (patients: $56.5 \pm 9.6$ years; controls: $55.1 \pm 12.5$ years), and handedness ( 9 right hand dominant, 2 left hand dominant in each group). All subjects gave informed consent to the experimental protocols approved by the Johns Hopkins institutional review board. Seven patients were diagnosed with a genetically defined spinocerebellar ataxia (type 6, $n=6$; type $8, n=$ 1). Three patients presented with symptoms of sporadic cerebellar ataxia (sporadic), a disorder with pure cerebellar signs but no familial history. One patient presented with symptoms of autosomaldominant cerebellar ataxia type III, an inherited disorder with pure cerebellar signs but no definite genetic testing. There was no evidence of atrophy to the brainstem, white matter damage, or spontaneous nystagmus among these patients. The severity of movement impairment was quantified with the International Cooperative Ataxia Rating Scale (ICARS; Trouillas et al., 1997). Patients with greater impairments receive higher scores. The ICARS is divided into four categories: walking and balance, limb control (kinetic), speech, and eye movement. Eight of the patients exhibited saccadic dysmetria; the three least impaired subjects (based on total ICARS scores) did not. None of the patients included in this study had sensory loss assessed by clinical measures of proprioception and tactile sensation (Campbell, 2005). These clinical measures were performed while subjects were instructed to remain passive. Previous work has shown that individuals with these cerebellar diseases and no other clinical neurological impairments can have normal passive proprioception even when assessed quantitatively (Maschke et al., 2003; Bhanpuri et al., 2012). We corroborated the same passive proprioceptive findings in the present study. It should be noted that a total of 15 cerebellar patients and 12 control subjects were initially recruited for this study, but four patients and one control subject were eventually excluded from the final analysis for two reasons. First, three of the more impaired patients (ICARS $>40$ ) and one control subject were unable to properly modulate their movement velocity in the active proprioception task (see Materials and Methods for details). Second, one subject with an unidentified cerebellar syndrome presented with reduced tactile sensation and impaired proprioception and thus was excluded from the study. It is likely that this subject had extracerebellar lesions in addition to cerebellar damage.
Apparatus. Subjects' perceptual thresholds on the dominant arm were measured using the KINARM exoskeleton robot system (BKIN Technologies; Scott, 1999). Their arms rested in trays chosen to minimize skin compression and avoid activating cutaneous mechanoreceptors (Maschke et al., 2003; Bhanpuri et al., 2012). The shoulder was fixed at $30^{\circ}$ flexion and the elbow was free to move. Elbow angle was defined as the angle between the upper arm and forearm (Fig. 1A). Subjects could not see their arms and wore headphones playing white noise.

EMG recordings. EMG recordings were collected from the brachioradialis, biceps brachii, and triceps brachii of the tested arm using surface electrodes (Bagnoli EMG system; Delsys). The signals were amplified by 10,000 and sampled at $1 \mathrm{kHz}$. For the passive task (Task 1 ), trials with muscle activity above baseline were discarded (typically $\leq 5 \%$ of trials).

Procedure. Subjects performed three randomly ordered tasks that assessed passive proprioception (Task 1), active proprioception with simple dynamics (Task 2), and active proprioception with complex dynamics (Task 3). All three tasks followed a two-alternative forced-choice protocol (Gescheider, 1997). In Task 1 (passive discrimination), subjects were instructed to relax their arm and be passive. Each trial consisted of two subtrials during which the robot rotated the elbow to a different extent. More specifically, at the start of each subtrial, the visual display background turned red and the subject's forearm was rotated to the start position $\left(75^{\circ}\right)$ where a blue dot appeared. After a random delay (1-2 s), the display background changed to green, one or two bars appeared in the corner to indicate the first or second subtrial, respectively, and the forearm was rotated in the direction of flexion. The velocity was ramped up over $250 \mathrm{~ms}$ to a steady value of $5 \% \mathrm{~s}(\mathrm{SD} 0.05 \% \mathrm{~s}$ ) until the desired end position was reached (Fig. $1 D, E$, first column). We implemented a proportional-integral-derivative control algorithm to ensure smooth and accurate movements (Ellis, 2004). The arm was held at the end target for a random delay $(1-3 \mathrm{~s})$. After the first subtrial, the display changed to red and the arm was reset to the start position. After the second subtrial, a diamond appeared to indicate the end of the trial. Subjects then verbally stated "one" or "two" depending on which subtrial they perceived that the forearm moved a larger distance. The experimenter recorded the response and the robot reset the arm to the start position for the next trial.

The angular distance of each forearm movement was determined based on a modified staircase method (Arezzo et al., 1985; Gescheider, 1997). Two staircase sets were performed; the first to measure the upper threshold (UT) and the second to measure the lower threshold (LT). During the UT set, the standard was always a $10.00^{\circ}$ movement and the comparison ranged from $14.00^{\circ}$ to $10.10^{\circ}$ (specifically: $14.00^{\circ}, 13.50^{\circ}$, $13.00^{\circ}, 12.50^{\circ}, 12.00^{\circ}, 11.75^{\circ}, 11.50^{\circ}, 11.25^{\circ}, 11.00^{\circ}, 10.75^{\circ}, 10.50^{\circ}$, $10.30^{\circ}$, or $10.10^{\circ}$ ) and started at $13.00^{\circ}$. The order of the standard and comparison was randomized on every trial. If the subject responded correctly at the end of a trial, for the next trial, the comparison magnitude was shifted incrementally toward the standard; otherwise, the comparison was shifted away from the standard. This process continued until the subject made a total of five errors. For the LT set, the standard was $10.00^{\circ}$ and the comparison ranged from $6.00^{\circ}$ to $9.90^{\circ}$ (specifically: $6.00^{\circ}, 6.50^{\circ}$, $7.00^{\circ}, 7.50^{\circ}, 8.00^{\circ}, 8.25^{\circ}, 8.50^{\circ}, 8.75^{\circ}, 9.00^{\circ}, 9.25^{\circ}, 9.50^{\circ}, 9.70^{\circ}$, or $9.90^{\circ}$ ) and started at $7.00^{\circ}$. For all three tasks, before the UT and LT sets, a practice set was administered with comparison magnitudes of either $6.00^{\circ}$ or $14.00^{\circ}$ and five consecutive correct responses were required before proceeding.

Task 2 (active-simple discrimination) was similar to Task 1 with a few notable differences (Fig. $1 C$ ). First, subjects produced movement themselves. These self-driven movements were stopped at a predetermined distance unknown to the subject using robot-applied torques to create a "virtual wall" and "valley" that retained the subjects' arm at a specific location once it was reached (Fig. $1 D, E$, second column). Second, to limit impact when contacting the virtual wall during Task 2 (and Task 3 described below) and to promote similar movement patterns across subjects and tasks, a yellow bar (located below the subtrial indicator) was displayed for movements when the velocity at any point during the movement exceeded $15 \%$ and subjects were instructed to reduce movement speed. (In pilot studies, we found this to be a salient cue for guiding subjects to move with a mean velocity 
A

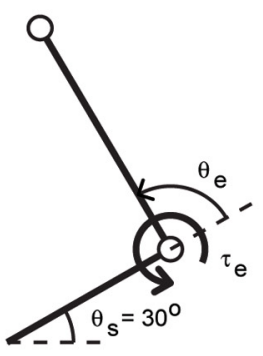

B

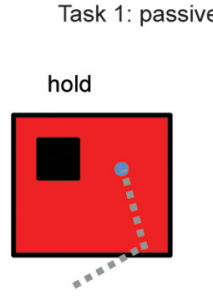

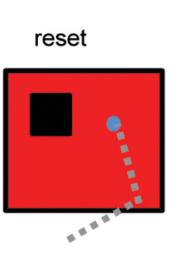
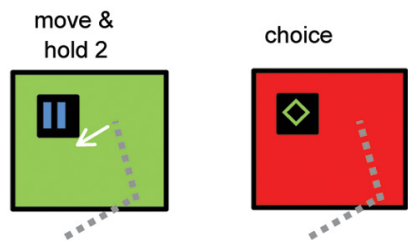

C Tasks 2 \& 3: active-simple and active-complex discrimination

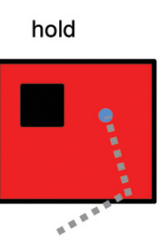

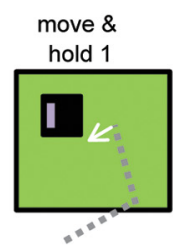

move to wall 1

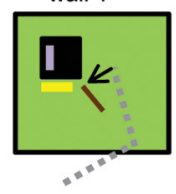

reset

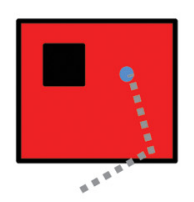

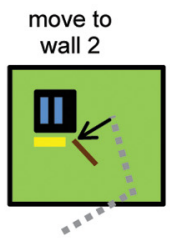

choice

time

Control

D
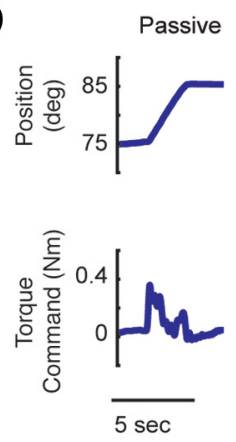

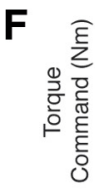

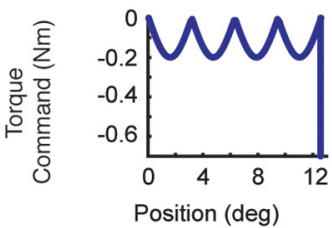

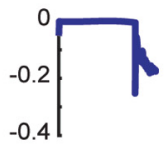

Active Simple<smiles>CCCC(C)(C)S(=O)(=O)c1ccccc1</smiles>

$-0.4$<smiles>C1CC2CCC2C1</smiles>
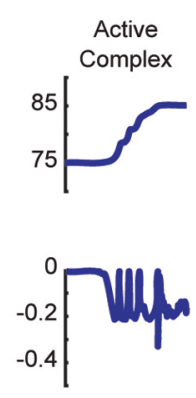

E
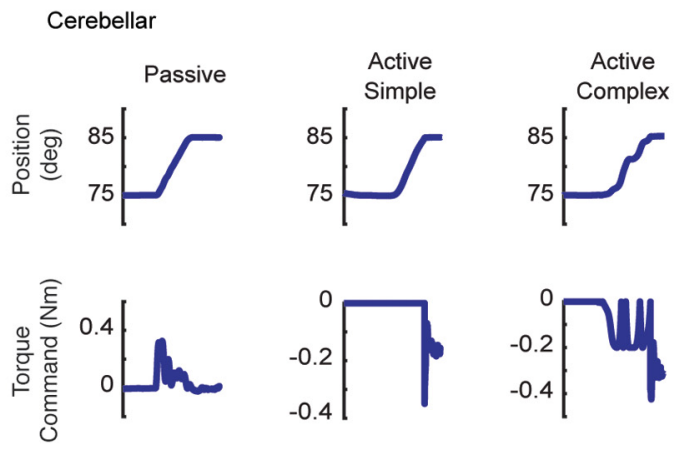

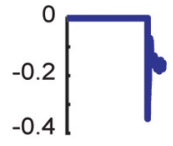

Figure 1. Task description and example trials. $\boldsymbol{A}, 0$ verhead view of arm schematic denoting conventions used to report elbow angle $\left(\theta_{\mathrm{e}}\right)$ and applied torque about the elbow $\left(\tau_{\mathrm{e}}\right)$. The shoulder angle $\left(\theta_{\mathrm{s}}\right)$ was fixed with a mechanical clamp at $30^{\circ}$. B , Task 1: Passive discrimination. Subjects were instructed to remain passive throughout the task. At the start of each trial, the robot moved the arm to the start position $\left(\theta_{\mathrm{e}}=75^{\circ}\right)$. Next, the arm was moved a specified distance and briefly held at the end location. The arm was returned to the start position and then moved a different distance. Subjects were asked to report whether Movement 1 or Movement 2 (indicated by bars in top left corner) went farther. White arrows indicate passive, robot-driven movement. C, Tasks 2 and 3: Active discrimination. Similar to Task 1 with the exception that, during the movement phase, subjects actively moved until the robot halted the arm at a specified location with a virtual wall (brown line, which is illustrative only and was not shown to subjects). Black arrows indicate active, subject-driven movement. The yellow horizontal bar was shown only if angular velocity exceeded $15 \%$. The dotted lines representing the arm are for illustrative purposes only, as subjects could not see their arms in either of the tasks. $\boldsymbol{D}-\boldsymbol{E}$, Example $10^{\circ}$ movement trials for control (D) and cerebellar (E) subjects. The elbow angle (top) and commanded robot torque (bottom) are shown for the passive task (first column), active-simple task (second column), and active-complex task (third column). Horizontal scale bar represents $5 \mathrm{~s}$. $\boldsymbol{F}$, Position-based torque command pattern for a $12.25^{\circ}$ trial for the active-complex task. All active-complex trials imposed two consecutive rectified sine waves of resistive torque as a function of distance from the start position.

near $5 \%$ s). Three patients and one control were unable to correctly modulate their movement velocity (instantaneous velocity $>15 \%$ in $>10 \%$ of trials) and were excluded from analysis.

Task 3 (active-complex discrimination) was the same as Task 2 except that, during movement, the robot produced a "complex pattern" of resistive (extension) torque (Fig. 1D,E, third column). The resistive torque, $\tau$, produced for a trial of total angular distance $\theta_{\mathrm{T}}$, was a function of the angular position, $\theta$ :

$$
\tau(\theta)=-0.2\left|\sin \left(\frac{4 \pi \theta}{\theta_{\tau}}\right)\right|
$$

This resulted in position-based virtual "bumps" that subjects traversed before encountering the virtual wall and valley (Fig. $1 F$ ). The pattern of external torque depended on $\theta_{\mathrm{T}}$ and thus was difficult to predict. All three tasks used the same distances for the standard and comparison movements (listed above).

Measurements and analysis. UT and LT were computed using established methods for processing information collected from staircase procedures (Arezzo et al., 1985; Gescheider, 1997). Each staircase set concluded after the subject made five errors (i.e., incorrectly identified the longer subtrial). The comparison distances for the five error trials and the five correctly identified trials closest to the standard distance were combined into a single array. The longest and shortest distances were removed to avoid potential outliers. The remaining eight values were averaged to compute each threshold. The point of subjective equality (PSE) was the average of the UT and LT. These values were used to compute the Weber fraction (WF): 


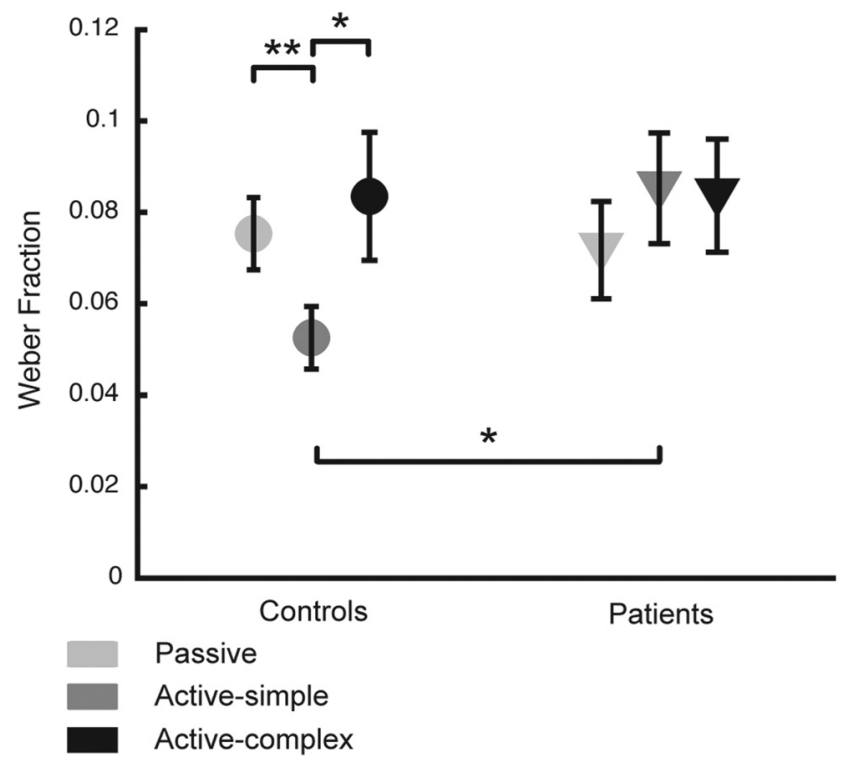

Figure 2. Comparison of WFs. Error bars indicate SEM. The only significant difference across groups for the three tasks was for the active-simple task $(p<0.031)$. Controls $(n=11)$ were significantly different at the active-simple task compared with the passive task $(p<0.005)$ and the active-complex task $(p<0.022)$, but they did not show a difference between the passive and active-complex tasks $(p>0.38)$. Among the patients $(n=11)$, there were no significant differences between tasks (all $p>0.28$ ). ${ }^{*} p<0.05,{ }^{* *} p<0.005$.

$$
\mathrm{WF}=(\mathrm{UT}-\mathrm{LT}) /(2 \times \mathrm{PSE})
$$

The WF reflects perceptual sensitivity, where lower WFs indicates better discrimination between two stimuli (Gescheider, 1997). Repeated-measures ANOVA ( 3 tasks $\times 2$ subject groups) was performed on the WFs and PSEs. Post hoc within-group comparisons of WFs were conducted using a $t$ test for dependent samples and differences across groups were assessed with a $t$ test for independent samples. In addition, Wilcoxon signed-rank tests (nonparametric tests for matched pairs) were performed on individual subject UT and LT data to determine whether differences between tasks reached statistical significance.

We also investigated several aspects of how subjects moved during the tasks. We were particularly concerned that subjects moved similarly for the active task, because differences in movement would be a potential confound. For a given subject and task, we computed the average values across all trials for maximum robot torque $(\mathrm{Nm})$, mean velocity $(\%)$, and smoothness index $\left({ }^{\circ}\right)$. Smoothness index was defined as the SD of the high-pass-filtered (second-order, $2 \mathrm{~Hz}$ cutoff, Butterworth) position data (low values indicate smooth movement). The individual subject means were averaged across groups for comparison. Data analysis was performed using MATLAB (MathWorks).

\section{Results}

The responses from the modified staircase method were used to compute the UT and LT, which in turn were used to compute the PSE and WF for a given subject and task. For example, in the passive task, the UT for one control subject was computed as $11.13^{\circ}\left(\mathrm{SE}=0.13\right.$; raw values: $10.50^{\circ}, 10.75^{\circ}, 11.00^{\circ}, 11.25^{\circ}$, $\left.\left.11.25^{\circ}, 11.25^{\circ}, 11.50^{\circ}, 11.50\right]\right)$ and the LT was computed as $9.46^{\circ}$ $\left(\mathrm{SE}=0.05\right.$; raw values: $9.25^{\circ}, 9.25^{\circ}, 9.50^{\circ}, 9.50^{\circ}, 9.50^{\circ}, 9.50^{\circ}$, $\left.\left.9.50^{\circ}, 9.70\right]\right)$, resulting in a PSE of $10.30^{\circ}$ and a WF of 0.081 . The group average of WFs ranged between $\sim 0.05$ and 0.09 (Fig. $2 B$ ), which means that, on average, the difference between the UT and LT ranged from $\sim 1^{\circ}$ to $\sim 1.8^{\circ}$ depending on the task (Equation $2)$. The average SE of UT and LT measurements across the different tasks was $0.09^{\circ}$ for both the control group and the patient group. This is $5-10 \%$ of the threshold differences, indicating that measurement resolution was sufficient for the tasks described in this study.

A repeated-measures ANOVA for WFs ( 3 tasks $\times 2$ subject groups) found a significant task-subject group interaction $(F=3.81, p<0.031)$ but no significant effect of task $(F=$ $2.20, p>0.12)$ or group $(F=0.54, p>0.47)$. Post hoc $t$ tests explored in greater detail the differences in performance underlying the interaction. As seen in previous studies (Maschke et al., 2003; Bhanpuri et al., 2012), passive proprioception (Task 1, no EMG activity) was similar between groups, as indicated by no difference in WFs (Fig. 2, $t=-0.25, p>0.80$ ). Controls demonstrated better precision of proprioception in the active-simple task (Task 2) compared with the passive task (Fig. 2, $t=3.67, p<0.005$ ), as expected (Adamovich et al., 1998; Fuentes and Bastian, 2010). However, their performance was not improved in the active-complex task (Task 3 ) - it was no different from the passive task (Fig. $2, t=-0.38, p>0.38$ ) and significantly worse than the active-simple task (Fig. 2, $t=$ $-2.73, p<0.022$ ). In contrast, the patient group showed no significant differences in WFs between tasks (Fig. 2; all $p>$ 0.28 ) and were significantly worse than controls at the activesimple task (Fig. 2, $t=-2.53, p<0.030$, control average: 0.053 ; patient average 0.085 ; a $60 \%$ difference). In summary, unlike controls, the patient group did not show an improvement in proprioceptive sensitivity during active movements with simple and predictable dynamics; however, they were similar to controls in passive proprioception and active proprioception when the movement dynamics were complex and unpredictable.

On an individual basis, seven of 11 controls demonstrated an improvement of at least 0.015 in WF between the passive and active-simple task (i.e., $\mathrm{WF}_{\text {passive }}-\mathrm{WF}_{\text {active-simple }}>0.015$ ). Furthermore, all seven showed a statistically significant improvement in either the UT or LT according to a Wilcoxon signed-rank test (all $p<0.02)$. Only three of 11 patients showed an improvement of at least 0.015 from passive to active-simple (only two showed significant improvement in either UT or LT according to the Wilcoxon signed-rank test; all $p<0.04$ ). In sum, $64 \%$ of controls and only $18 \%$ of patients showed either a significant improvement in UT or LT between the passive and active-simple tasks that resulted in a WF change of at least 0.015 .

The only obvious characteristic distinguishing the three patients who showed improvement in WF (mentioned above) from the rest of the group is that their ICARS scores were lower (i.e., they were less motorically impaired) than all but two other patient's scores. However, across the entire patient group, we found no significant correlations between WF for the different tasks and ICARS scores (for both total ICARS and kinetic subscores, all were $p>0.4$ ).

We ruled out any differences in movement patterns between the two groups in the various conditions using three metrics: maximum torque, mean velocity, and smoothness index. The groups were similar for these parameters across all three tasks (Fig. 3; all $p>0.18$ ). In addition, none of these metrics was significantly correlated with the WFs for either group (all $p>$ 0.13 ). Although the patients had movement abnormalities during the neurological examination, they performed the tasks similarly to controls, probably because movements were at a single joint (Bastian et al., 2000) and slow (Topka et al., 1998). We also performed repeated-measures ANOVA on PSEs to determine whether there was a significant effect of group or task on the average of the UT and LT. We did not expect to see an effect here because we hypothesized that group and task would affect the UT and LT similarly. As anticipated, there were no significant effects of task $(F=$ 

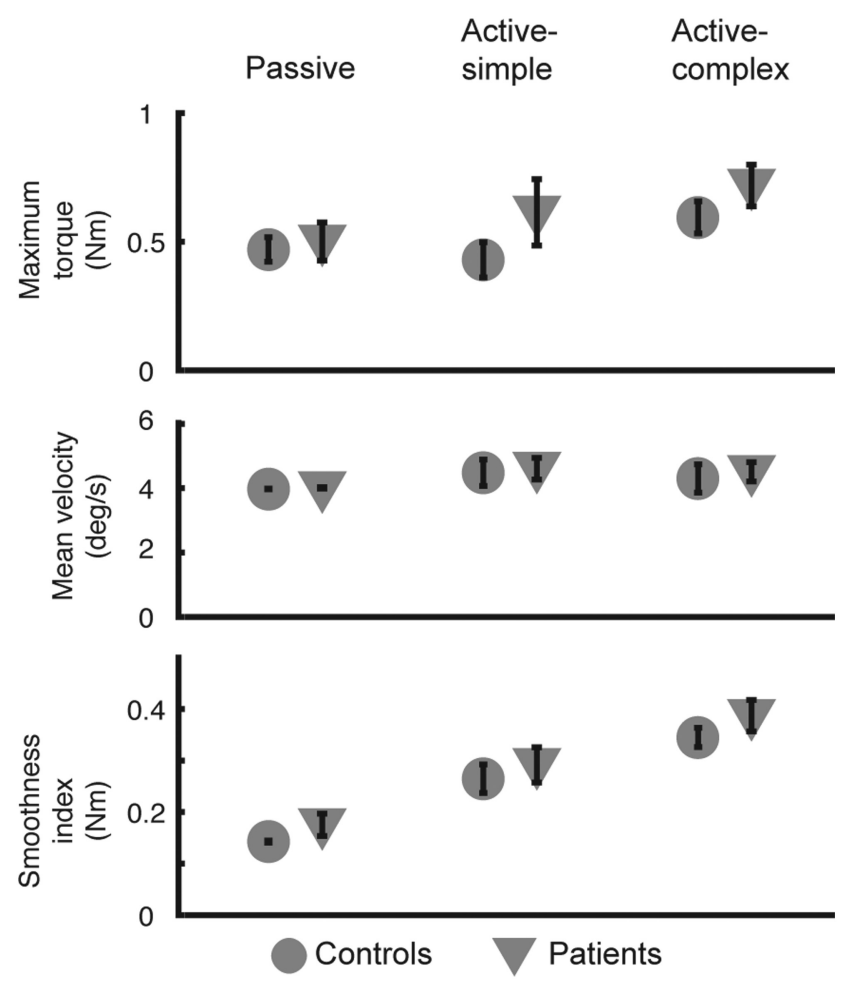

Figure 3. Behavioral comparison of controls and cerebellar patients. Possible differences in movement during tasks could account for differences in perception. Group average of average maximum robot torque (top row), average mean velocity (middle row), and average smoothness index (bottom row) for all tasks. Smoothness index was computed as the SD of the highpass-filtered (second-order, $2 \mathrm{~Hz}$ cutoff) position data (low values indicate smooth movement). Error bars indicate SE. Controls and patients demonstrated similar behavior (all $p>0.18$ ).

$0.88, p>0.35)$, group $(F=1.98, p>0.15)$, or task-group interaction $(F=0.16, p>0.85)$.

\section{Discussion}

Our results show that a cerebellum-dependent mechanism is used to improve active versus passive proprioception during a discrimination task. Cerebellar patients have the same precision as controls in passive proprioception, but whereas controls improve with active movement (Adamovich et al., 1998; Zia et al., 2000; Laufer et al., 2001; Fuentes and Bastian, 2010), patients do not. Importantly, we show that not all self-generated movement improves proprioception; when control subjects encounter unpredictable forces during movement, their active proprioceptive precision worsens to passive levels. Therefore, controls look very much like cerebellar patients when the movement outcome from their motor commands becomes unpredictable. We interpret these results to mean that the cerebellum is contributing to a prediction about the state of the limb given the motor commands being executed and that this can be used in combination with proprioceptive input to improve perception.

The current work extends beyond previous research on cerebellar contributions to perception (Angel, 1980; Synofzik et al., 2008; Bhanpuri et al., 2012) in a few critical ways. First, we identified a perceptual deficit that is limited to proprioception, whereas the other studies involved force perception (Angel, 1980; Bhanpuri et al., 2012) or vision (Synofzik et al., 2008 ) in addition to proprioception. Therefore, it is possible that the previously described deficits are due to fundamental problems in active proprioception and not due to combining proprioceptive information with different sensory modalities (i.e., force, vision). Second, an important difference between this study and the study conducted by Synofzik et al. (2008) is that the patients in this study exhibited perceptual deficits during familiar movements, whereas patients in the other the study only displayed perceptual abnormalities for a task involving a sensorimotor manipulation. Based on our results, we would have predicted that the patients studied by Synofzik et al. (2008) would have been worse (i.e., higher just noticeable differences) with regard to detecting manipulated visual feedback during preadaptation compared with controls, but perhaps the method used was not sensitive enough to uncover this distinction. Further testing should be done to resolve this difference. Third, we show that control subject perceptions can be perturbed and resemble patients by restricting movements to a novel dynamic environment. This provides evidence that one's ability to predict movement outcomes is necessary for normal perception during action. Furthermore, we suggest that studying healthy individuals in the active-complex condition can be a useful model for studying internal model deficits when access to cerebellar patients is limited.

There is evidence to suggest the cerebellum continuously computes state predictions based on efferent copies of the outgoing motor command to guide movement (Wolpert et al., 1998; Miall et al., 2007; Xu-Wilson et al., 2009). It is also well established that people can optimally integrate two sensory estimates to form a single, more precise estimate of their body state (e.g., hand position; van Beers et al., 1999; van Beers et al., 2002). Here, subjects' internal prediction of elbow movement could be combined with sensory information from the periphery to refine their final estimate of the forearm position after voluntary movement. These predictions are unavailable during passive movements and imprecise when movement dynamics are difficult to predict. In addition, when the cerebellum is damaged, we propose that the predictive internal models are much more variable and, therefore, according to optimal integration of two information streams (van Beers et al., 1999; Ernst and Banks, 2002; van Beers et al., 2002), the final percept would be based primarily on peripheral information.

We have uncovered a perceptual deficit among a patient population that has not been reported in the literature. Standard clinical tests for proprioceptive function are passive in nature: the examiner moves the limb or digit of a subject and then the subject reports the change in position either verbally or by matching with the contralateral body part (Campbell, 2005). Cerebellar damage does not impair this type of perception (Holmes, 1917; Maschke et al., 2003; Bhanpuri et al., 2012). With regard to the individuals with SCA6 specifically, signs of sensory neuropathy have varied in the literature. Two studies did not detect sensory neuropathy in these individuals using clinical sensory tests, nerve conduction studies, or somatosensory evoked potentials (Nagai et al., 1998; Lee et al., 2003), whereas another study observed mild impairments (Schöls et al., 1998). Based on the results of our clinical examinations and the passive proprioception experiment (which is more precise than typical clinical tests), we believe that the deficits we observed in active proprioception were not due to peripheral sensory dysfunction.

Perhaps most importantly, we have shown here that cerebellar patients are impaired at discriminating between limb positions after different active, self-driven movements. During natural behavior, our body state is modified primarily by active movements rather than passive movements, so it follows that the cerebellum is crucial to normal proprioception in most practical circumstances. Clinicians and therapists 
should take note that, in addition to obvious movement abnormalities, cerebellar patients have an impaired perception of movement outcomes, so interventions may need to consider proprioceptive deficits along with the classic symptoms of ataxia.

\section{References}

Adamovich SV, Berkinblit MB, Fookson O, Poizner H (1998) Pointing in 3D space to remembered targets. I. Kinesthetic versus visual target presentation. J Neurophysiol 79:2833-2846. Medline

Angel RW (1980) Barognosis in a patient with hemiataxia. Ann Neurol 7:73-77. CrossRef Medline

Arezzo JC, Schaumburg HH, Laudadio C (1985) The Vibratron: a simple device for quantitative evaluation of tactile/vibratory sense. Neurology 35:169.

Bastian AJ, Zackowski KM, Thach WT (2000) Cerebellar ataxia: torque deficiency or torque mismatch between joints? J Neurophysiol 83: 3019-3030. Medline

Bhanpuri NH, Okamura AM, Bastian AJ (2012) Active force perception depends on cerebellar function. J Neurophysiol 107:1612-1620. CrossRef Medline

Campbell WW (2005) DeJong's the neurologic examination. Baltimore: Lippincott Williams and Wilkins.

Ellis G (2004) Control system design guide: using your computer to understand and diagnose feedback controllers, Ed 3. San Diego: Academic.

Ernst MO, Banks MS (2002) Humans integrate visual and haptic information in a statistically optimal fashion. Nature 415:429-433. CrossRef Medline

Fuentes CT, Bastian AJ (2010) Where is your arm? Variations in proprioception across space and tasks. J Neurophysiol 103:164-171. CrossRef Medline

Gescheider GA (1997) Psychophysics: the fundamentals, Ed 3. Mahwah, NJ: Psychology Press.

Holmes G (1917) The symptoms of acute cerebellar injuries due to gunshot injuries. Brain 40:461-535. CrossRef

Jones KE, Wessberg J, Vallbo AB (2001) Directional tuning of human forearm muscle afferents during voluntary wrist movements. J Physiol 536: 635-647. CrossRef Medline

Laufer Y, Hocherman S, Dickstein R (2001) Accuracy of reproducing hand position when using active compared with passive movement. Physiother Res Int 6:65-75. CrossRef Medline

Lee YC, Chen JT, Liao KK, Wu ZA, Soong BW (2003) Prolonged cortical relay time of long latency reflex and central motor conduction in patients with spinocerebellar ataxia type 6. Clin Neurophysiol 114:458-462. CrossRef Medline

Maschke M, Gomez CM, Tuite PJ, Konczak J (2003) Dysfunction of the basal ganglia, but not the cerebellum, impairs kinaesthesia. Brain 126: 2312-2322. CrossRef Medline
Miall RC, Christensen LO, Cain O, Stanley J (2007) Disruption of state estimation in the human lateral cerebellum. PLoS Biol 5:e316. CrossRef Medline

Nagai Y, Azuma T, Funauchi M, Fujita M, Umi M, Hirano M, Matsubara T, Ueno S (1998) Clinical and molecular genetic study in seven Japanese families with spinocerebellar ataxia type 6. J Neurol Sci 157:52-59. CrossRef Medline

Paillard J, Brouchon M (1974) A proprioceptive contribution to the spatial encoding of position cues for ballistic movements. Brain Res 71:273-284. CrossRef Medline

Proske U, Gandevia SC (2009) The kinaesthetic senses. J Physiol 587:4139_ 4146. CrossRef Medline

Schöls L, Krüger R, Amoiridis G, Przuntek H, Epplen JT, Riess O (1998) Spinocerebellar ataxia type 6: genotype and phenotype in German kindreds. J Neurol Neurosurg Psychiatr 64:67-73. CrossRef Medline

Scott SH (1999) Apparatus for measuring and perturbing shoulder and elbow joint positions and torques during reaching. J Neurosci Methods 89:119-127. CrossRef Medline

Synofzik M, Lindner A, Thier P (2008) The cerebellum updates predictions about the visual consequences of one's behavior. Curr Biol 18:814-818. CrossRef Medline

Todorov E, Jordan MI (2002) Optimal feedback control as a theory of motor coordination. Nat Neurosci 5:1226-1235. CrossRef Medline

Topka H, Konczak J, Dichgans J (1998) Coordination of multi-joint arm movements in cerebellar ataxia. Exp Brain Res 119:483-492. Medline

Trouillas P, Takayanagi T, Hallett M, Currier RD, Subramony SH, Wessel K, Bryer A, Diener HC, Massaquoi S, Gomez CM, Coutinho P, Ben Hamida M, Campanella G, Filla A, Schut L, Timann D, Honnorat J, Nighoghossian N, Manyam B (1997) International Cooperative Ataxia Rating Scale for pharmacological assessment of the cerebellar syndrome. The Ataxia Neuropharmacology Committee of the World Federation of Neurology. J Neurol Sci 145:205-211. CrossRef Medline

van Beers RJ, Sittig AC, Gon JJ (1999) Integration of proprioceptive and visual position-information: an experimentally supported model. J Neurophysiol 81:1355-1364. Medline

van Beers RJ, Wolpert DM, Haggard P (2002) When feeling is more important than seeing in sensorimotor adaptation. Curr Biol 12:834837. CrossRef Medline

Wolpert DM, Ghahramani Z, Jordan MI (1995) An internal model for sensorimotor integration. Science 269:1880-1882. CrossRef Medline

Wolpert DM, Miall RC, Kawato M (1998) Internal models in the cerebellum. Trends Cogn Sci 2:338-347. CrossRef Medline

Xu-Wilson M, Chen-Harris H, Zee DS, Shadmehr R (2009) Cerebellar contributions to adaptive control of saccades in humans. J Neurosci 29: 12930-12939. CrossRef Medline

Zia S, Cody F, O’Boyle D (2000) Joint position sense is impaired by Parkinson's disease. Ann Neurol 47:218-228. Medline 